\title{
Evaluation of the head and neck cancer patient concerns inventory in a cohort of patients attending routine multidisciplinary speech and language therapy/dietitian follow up clinics
}

\author{
Annette C. Zuydam ${ }^{1 \wedge}$, Derek Lowe ${ }^{2}$, Simon N. Rogers ${ }^{3,4} \wedge$ \\ ${ }^{1}$ Shrewsbury and Telford NHS Trust, Shrewsbury, UK; ${ }^{2}$ Evidence-Based Practice Research Centre (EPRC), Faculty of Health, Edge Hill University, \\ Ormskirk, UK; ${ }^{3}$ Evidence-Based Practice Research Centre (EPRC), Faculty of Health and Social Care, Edge Hill University, Ormskirk, UK; \\ ${ }^{4}$ Regional Maxillofacial Unit, University Hospital Aintree, Liverpool, UK \\ Contributions: (I) Conception and design: AC Zuydam; (II) Administrative support: AC Zuydam; (III) Provision of study materials or patients: AC \\ Zuydam; (IV) Collection and assembly of data: AC Zuydam; (V) Data analysis and interpretation: D Lowe; (VI) Manuscript writing: All authors; (VII) \\ Final approval of manuscript: All authors. \\ Correspondence to: Annette C. Zuydam, BSc (Hons), MSc. Macmillan Speech and Language Therapist, Macmillan Integrated Therapy Service, Admin \\ Corridor, Royal Shrewsbury Hospital, Mytton Oak, Shrewsbury SY3 8XQ, UK. Email: annette.zuydam@nhs.net.
}

Background: Speech and language therapy (SLT) and Dietetics follow up clinics following head and neck
cancer (HNC) treatment are an integral part of multidisciplinary support. The use of a condition specific
prompt list can help patients to raise issues, that otherwise might be missed. The objective of the present
study was to evaluate the Patient Concerns Inventory-Head and Neck (PCI-HN) in a combined SLT/
dietitian (Diet) clinic, as an adjunct to routine care. Methods: This prospective observational study recruited patients from $30^{\text {th }}$ January 2019 to $19^{\text {th }}$ March 2020 across one trust involving two SLTs and two Diets. Patients completed the PCI-HN and a single quality of life (QOL) question prior to their consultation.

Results: Ninety-four patients were recruited from a sample of 100 consecutive patients, and they first used the PCI-HN at a median (IQR) of 2.4 (1.3-7.1) months after the end of treatment. The PCI-HN was used more than once by 65 patients. When first used, the most common selections were dry mouth $(62 \%)$, chewing/eating (55\%), mucus (44\%), swallowing (41\%), appetite (35\%), fatigue/tiredness (34\%), energy levels (33\%), coughing (28\%), weight (28\%), speech/voice/being understood (27\%) and taste (27\%). PCI$\mathrm{HN}$ items varied by tumour site, overall clinical stage, and primary treatment. Ninety one percent (32/35) of patients said the PCI-HN "definitely" helped communication with the clinician and $83 \%$ (29/35) "definitely" wanted to continue using it.

Conclusions: Our preliminary data suggest that the PCI-HN is suitable for use in routine clinical practice in combined SLT and Diet clinics, as an adjunct to routine care. It enabled a patient led approach to the consultation, addressing concerns, and identifying interventions to support patients through their recovery. Early detection of issues which impact on eating, drinking and communication, allows the opportunity for the identification, and provision, of Speech and Language Therapy and Dietetic interventions, which can aid adaption, and ultimately, promote better outcomes.

Keywords: Speech and language therapy (SLT); dietitian (Diet); head and neck cancer (HNC); follow up clinics; patient concerns inventory

Received: 02 August 2021; Accepted: 10 December 2021; Published: 10 March 2022.

doi: 10.21037/fomm-21-81

View this article at: https://dx.doi.org/10.21037/fomm-21-81

^ ORCID: Annette C. Zuydam, 0000-0002-2652-6209; Simon N. Rogers, 0000-0002-5989-6142. 


\section{Introduction}

Treatment for head and neck cancer (HNC) can result in very significant functional impairments often involving difficulty in communication and swallowing, which are associated with poorer health related quality of life (HRQOL) (1). Speech and language therapists (SLTs) and dietitians (Diets) are key members of the Multidisciplinary team (MDT), and an important part of their role is to help optimize clinical, functional and HRQOL outcomes for their patients (2). MDT combined, SLT and Diet rehabilitation clinics post treatment provide opportunities to focus on role specific issues, to identify early concerns, and to provide interventions to support patient recovery. However, it can be difficult to elicit unmet supportive care needs (3). Various patient reported outcome measures (PROMS) have been used to evaluate swallowing, voice, and speech function following HNC treatment $(4,5)$. These are often questionnaire based, can be time consuming to complete, and they cannot always capture adequately the wide range of physical, psychological, and social factors which impact on communication, swallowing and nutrition. There are a number of objective assessments, of varying complexity, available to clinicians, such as weight, body mass index, video fluoroscopy and speech intelligibility. However, these measurements of functional outcome do not necessarily correlate directly with HRQOL on an individual patient basis and can miss the patient's perspective of a poor outcome. Patients value the chance to discuss their concerns (6), and there are various tools for helping to identify unmet needs in the HNC setting (7). The Patient Concerns Inventory-Head and Neck (PCI-HN) is a condition-specific item prompt list (8), which patients select from before their appointment, to help guide the outpatient consultation. The PCI-HN comprises of 56 items that cover concerns in relation to sequelae of treatment. It helps to focus the consultation (9), aid clinicianpatient communication (10), provide clinical benefit (11), and is simple enough to use in routine practice (12). The PCI-HN has been recommended in a systematic review with particular emphasis on the importance of content validity over quantitative psychometric properties (7).

The PCI-HN was developed for use in consultant led HNC clinics. However, it covers several treatment issues relating to SLT and Diet interventions, with the PCI-HN items chewing/eating, swallowing, speech/voice/being understood, being among the ten most common issues raised by patients $(13,14)$. It also incorporates several wider issues commonly reported by patients following treatment, such as dry mouth and taste after (chemo-) radiotherapy which have been found to be symptom drivers of oral intake (15-17). Fatigue is also an important issue as it can contribute to low mood, which compounds swallowing problems, and can, in turn, affect appetite, nutrition and weight loss (18). The PCI-HN has been used as a one-page prompt sheet (14), and the advantage of this approach is that it offers a simple means of ensuring that issues patients want to discuss at their consultation are not missed.

Various cohorts have been published demonstrating the value of the PCI-HN in the consultant clinic setting (7-12). However, to the best of our knowledge, it has not been evaluated to determine if there are additional benefits to practice in other MDT clinical settings. We believe that this is the first time the PCI-HN has been reported in patients attending a routine combined SLT/Diet post treatment rehabilitation out-patient clinic.

In our centre, all patients are referred post treatment to a combined SLT/Diet clinic. The purpose of the clinic is to provide integrated and timely intervention in relation to communication, swallowing and nutrition, and other treatment related issues, and to support them through their recovery. In our experience, patients also frequently raise wider issues which impact on their quality of life (QOL). We hypothesised, therefore, that the PCI-HN was a tool that could be used in our clinic to guide the consultation. In our centre, patients are seen early post treatment, and this provides an ideal opportunity for the clinicians to address concerns and, where appropriate, signpost to other services.

The objective of the current study was to evaluate the PCI-HN in the setting of a combined, post treatment SLT/ Diet clinic, as an adjunct to routine care.

We present the following article in accordance with the STROBE checklist (available at https://fomm.amegroups. com/article/view/10.21037/fomm-21-81/rc).

\section{Methods}

Patients were recruited prospectively from $30^{\text {th }}$ January 2019 to $19^{\text {th }}$ March 2020 when attending face to face SLT/ Diet post treatment rehabilitation clinics across two clinics in one trust, involving two SLT and two Diets. All post treatment patients were referred to the clinic, and patients were eligible for the study if diagnosed with HNC and treated with surgery, chemotherapy and/or radiotherapy with curative intent. Those with cognitive impairment or psychiatric disorders, and those with recurrence were excluded. 
The PCI-HN consists of 56 items grouped into domains: (I) physical and functional well-being (29 items); (II) treatment-related (4 items); (III) social care/social well-being (9 items); (IV) psychological and emotional well-being/spiritual (14 items) (9). Patients were asked to complete the paper version of the PCI-HN on arrival at clinic, by therapists in one clinic and by reception in the other, with the completed paper being used in the consultation. There was also a single question on the paper about overall QOL, (outstanding, very good, good, fair, poor, very poor), and patients were asked to consider not only physical and mental health, but also other factors, such as family, friends, spirituality, or personal leisure activities that were important to their enjoyment of life. The prompt sheet has previously been used in an international PCIHN study (14). Patients were asked to use it whenever they presented for review within the follow-up period.

An evaluation of the PCI-HN was incorporated from August 2019, comprising a paper-based feedback that asked patients 5 questions (each on a 5 point Likert scale) covering (I) how easy it had been to complete the PCI-HN (very easy, easy, neither easy nor difficult, difficult, very difficult); (II) whether a problem was caused in the running of their appointment (not at all, a little, somewhat, a great deal, not sure); (III) how much the PCI-HN items they selected were included in their consultation (definitely yes, maybe yes, not sure, maybe not, definitely not); (IV) whether use of the PCI-HN helped them communicate with the clinician (definitely yes, maybe yes, not sure, maybe not, definitely not); and (V) whether they would like to continue using the PCI-HN in future appointments (definitely yes, maybe yes, not sure, maybe not, definitely not).

\section{Statistical analysis}

The Mann-Whitney (2 comparison groups) test or the Kruskal-Wallis ( $>2$ groups) test was used to compare patient groups (gender, age, tumour site, overall clinical stage, primary treatment) in the total number of PCI-HN items selected and in the number of items selected in the physical and functional wellbeing domain. Fishers exact test was used to compare patient groups in selecting one or more items from each of the other three PCI-HN domains, in regard to selecting specific PCI-HN items and regarding overall QOL. Spearman's Rho was used to measure correlation between overall QOL (6-point scale) and the total number of PCI-HN items. McNemar's test was used to assess the change between first and second PCI-HN occasions in the percentage of patients reporting a good or better overall QOL. SPSS version 25 was used for all analyses and graphics.

\section{Ethical statement}

The study was conducted in accordance with the Declaration of Helsinki (as revised in 2013). The data for this study collected were collected during routine follow up HNC clinics. The present study was granted local audit approval by the Shrewsbury and Telford Hospital Trust audit department but did not require formal IRAS ethics approval. Informed consent was taken from all individual participants.

\section{Results}

Ninety-four patients were recruited from a sample of 100 consecutive patients, and they first used the PCI-HN between $30^{\text {th }}$ January 2019 and $19^{\text {th }}$ March 2020 at a median (IQR) of 2.4 (1.3-7.1) months after the end of treatment. Median (IQR) age was 65 [60-72] years and 68\% [64] were male; other patient characteristics are shown in Table 1. Recruitment and follow up was stopped in March 2020 due to COVID 19.

The 94 patients used the PCI-HN on 228 occasions during the study period, with 3 patients refusing to use it on 5 occasions; 29 patients used it once only, 34 twice, 13 thrice and 18 on four to nine occasions. The PCI-HN was thus used more than once by 65 patients with the second PCI$\mathrm{HN}$ being a median (IQR) of 1.6 (1.2-2.3) months, range 0.3-11 after the first and the 69 subsequent occasions being a median (IQR) of 5.5 (3.5-7.4) months after the first, range 2-12 months. After completing one PCI-HN, 4 patients were lost to the study because of death (1) or recurrence (3). When first used, the most common selections (Figure 1) were dry mouth (62\%), chewing/eating (55\%), mucus (44\%), swallowing (41\%), appetite (35\%), fatigue/tiredness (34\%), energy levels (33\%), coughing (28\%), weight (28\%), speech/ voice/being understood (27\%) and taste (27\%). The group of items most selected initially were also the items most selected in repeat visits, though the frequency of selection was reduced (Table 2). The largest reductions were in selections of chewing/eating, mucus, coughing, swallowing and energy levels.

The median (IQR) total number of PCI-HN items initially selected was 7 [5-10], dominated (Table 3) by items from the physical \& functional well-being domain, median 
Table 1 Patient characteristics

\begin{tabular}{|c|c|c|}
\hline Characteristics & No. of patients & $\%$ \\
\hline Total & 94 & 100 \\
\hline \multicolumn{3}{|l|}{ Gender } \\
\hline Female & 30 & 32 \\
\hline Male & 64 & 68 \\
\hline \multicolumn{3}{|l|}{ Age at baseline } \\
\hline$<65$ & 44 & 47 \\
\hline$\geq 65$ & 50 & 53 \\
\hline \multicolumn{3}{|l|}{ Tumour site } \\
\hline Oral cavity & 19 & 20 \\
\hline Oropharynx & 47 & 50 \\
\hline Larynx & 15 & 16 \\
\hline Other** & 13 & 14 \\
\hline \multicolumn{3}{|l|}{ Diagnosis } \\
\hline $\operatorname{scC}$ & 87 & 93 \\
\hline Non-SCC & 7 & 7 \\
\hline \multicolumn{3}{|l|}{ Overall clinical stage } \\
\hline Advanced 3-4 & 66 & 76 \\
\hline Early 1-2 & 21 & 24 \\
\hline Not known & 7 & - \\
\hline \multicolumn{3}{|l|}{ Primary treatment } \\
\hline Surgery only & 14 & 15 \\
\hline RT only & 28 & 30 \\
\hline RT \& CT only & 41 & 45 \\
\hline Surgery \& (RT or RT\&CT) & 9 & 10 \\
\hline Not known & 2 & - \\
\hline \multicolumn{3}{|l|}{ Free-flap } \\
\hline Yes $^{*}$ & 17 & 18 \\
\hline No & 76 & 82 \\
\hline Not known & 1 & - \\
\hline
\end{tabular}

*, radial forearm [10], anterolateral thigh [3], scapula [2], fibula [1], pectoralis major [1]; **, parotid [6], metastatic lymph node/ unknown primary [5], follicular lymphoma post nasal space [1], piriform fossa [1]. RT, radiotherapy; CT, chemotherapy; SCC, squamous cell carcinoma.

(IQR) 6 [4-8]. This dominance was noted across all levels of the measured casemix, apart from a lesser dominance for patients having oral cavity tumours and for patients being treated with surgery alone. In these patients there was a higher proportion selecting items from the social and psychological domains. In terms of specific items commonly selected when the PCI-HN was first used (Table 4), a higher proportion of patients with oral cavity tumours selected speech/voice issues and conversely a higher proportion of patients with tumours located elsewhere selected fatigue and energy levels. A similar trend was seen for patients treated only by surgery in selecting speech/voice issues and for patients treated by radiotherapy +/- chemotherapy selecting fatigue and energy level items. Twelve of the 19 with oral cavity tumours were treated by surgery alone and 34 of the 45 with oropharyngeal tumours were treated with radiotherapy and chemotherapy without surgery. A higher proportion of patients with advanced stage tumours selected dry mouth and weight issues. Other notable associations not shown in Table 4, and at $\mathrm{P}<0.01$, were seen for mouth opening with tumour site (7/19 oral vs. 4/47 oropharynx vs. $0 / 15$ larynx vs. $0 / 13$ other, $\mathrm{P}=0.001$ ), percutaneous endoscopic gastrostomy (PEG) tube with age group (15/44 under 65 vs. $5 / 49$ over $65, \mathrm{P}=0.006$ ) and with tumour site $(1 / 19$ vs. $17 / 47$ vs. $2 / 15$ vs. $0 / 13, \mathrm{P}=0.003)$, and fears of the cancer returning with tumour site $(10 / 19$ vs. $6 / 47$ vs. $2 / 15$ vs. 2/13, $\mathrm{P}=0.005)$.

Overall QOL, was stated as "very good" or "outstanding" by $19 \%$ (17/91), "good" by 34\% [31], "fair" by 33\% [30] and "poor" or "very poor" by $14 \%$ [13]. In 62 patients using the PCI-HN a second time and reporting overall QOL on both occasions the percentage reporting a good or better QOL rose from 47\% [29] to 66\% [41], $\mathrm{P}=0.01 \mathrm{McNemar}$ test. There were no significant associations of casemix with overall QOL being good or better on the first occasion, nor with change between first and second occasions (Appendix 1). Overall QOL (six-point scale) was associated with the total number of PCI-HN items selected, Spearman Rho $=0.47$, $\mathrm{P}<0.001, \mathrm{n}=91$, Figure 2.

From August 2019 there were 35 evaluation forms completed by 33 patients, 16 after the first PCI-HN and 19 after a subsequent use. The PCI-HN was said by $66 \%$ $(23 / 35)$ to be "very easy" to complete (63\% first, $68 \%$ subsequent) with the remainder saying it was "easy", 94\% $(33 / 35)$ that it was "not at all" a problem in the running of appointments, 91\% (32/35) "definitely yes" the PCI$\mathrm{HN}$ items were discussed in consultations, 91\% (32/35) "definitely yes" the PCI-HN helped communication with the clinician and $83 \%$ (29/35) "definitely yes" they wanted to continue using the PCI-HN. Apart from two patients saying there had been "a little" bit of a problem in the running of their appointments there were no negative 


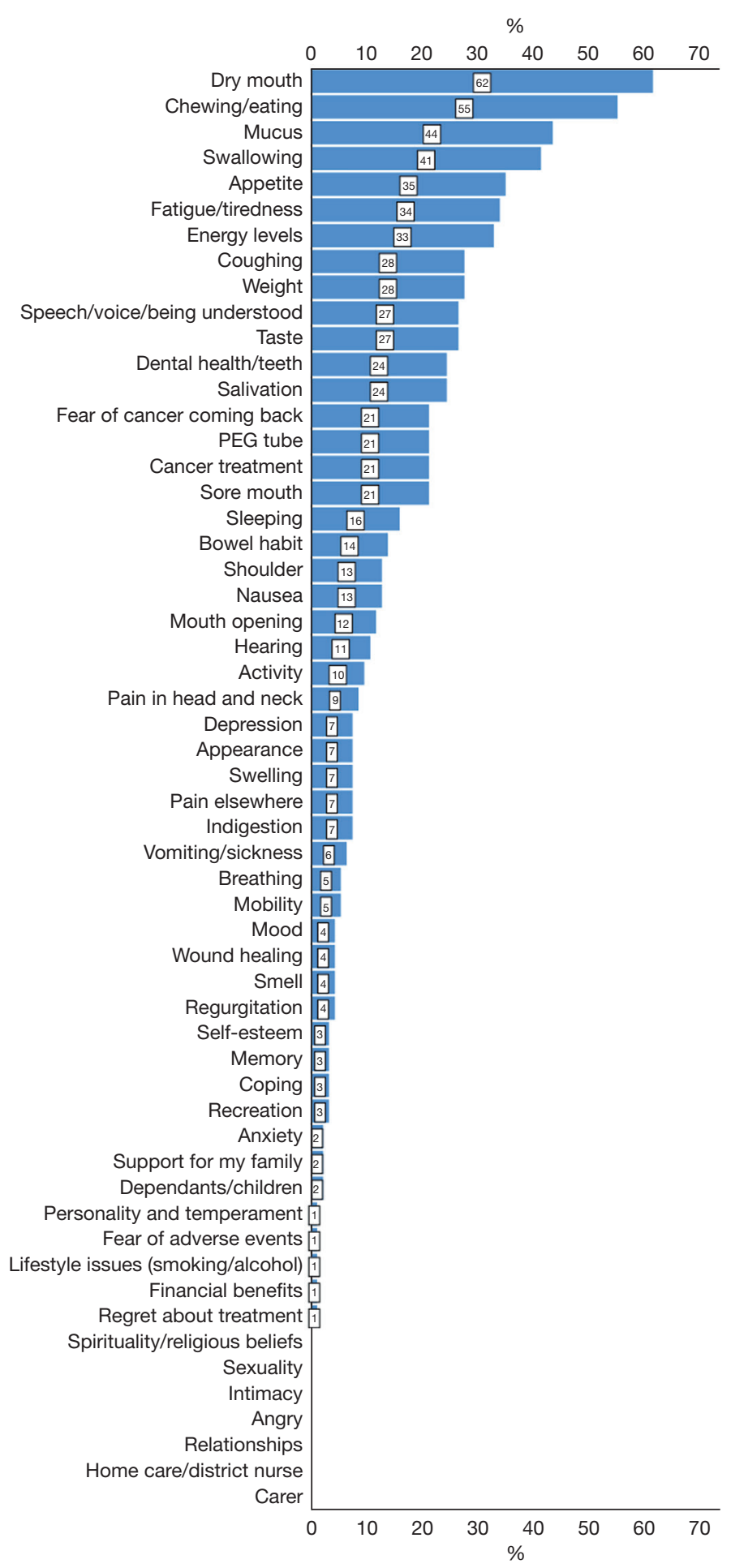

Figure 1 PCI items selected by 94 patients using the PCI for the first time. PCI, Patient Concerns Inventory. 
Table 2 PCI items most selected

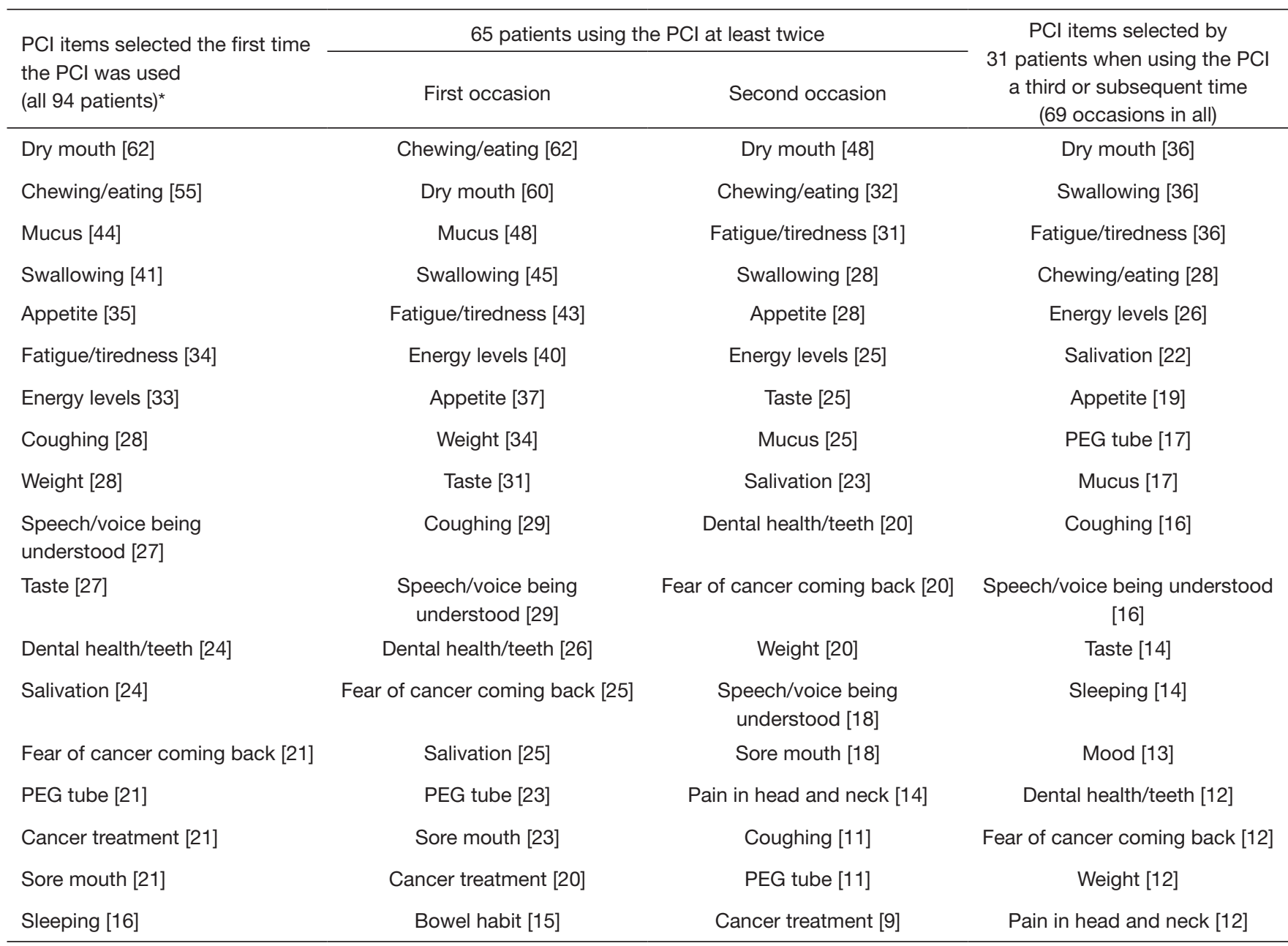

The results are presented as percentages. *, see Figure 1 for full list. PCl, percutaneous coronary intervention; PEG, percutaneous endoscopic gastrostomy.

responses across all 5 questions.

\section{Discussion}

SLT and Dietetic review following HNC is an integral part of multidisciplinary support. It provides a point of reference to discuss and assess treatment outcomes, give advice, and deliver interventions to aid recovery. The use of the PCI-HN in the consultant clinic setting has been validated in a number of studies (7-12). However, to the best of our knowledge, this is the first time the PCI-HN has been reported in the context of patients attending a routine SLT/Diet post treatment rehabilitation out-patient clinic. Consecutive patients helped to reduce non-responder bias and 94\% participated.
Only 3 of the 94 refused to use the PCI on 5 occasions and this seemed to be an issue of personal preference rather than any specific clinical characteristics. It is important to respect patients' choice in care wherever possible and the fact that so many choose to complete the PCI is a positive aspect to the prompt list approach. The PCI-HN single paper sheet has validity, having been used in a much larger international study (14). The PCI-HN form was completed on arrival at clinic, before the appointment, reflecting the situation in routine clinical practice. The timing post treatment was variable and some of the patients completed it multiple times. However, this was just a question of the natural passage of time, with earlier patients more likely to be seen more often. The single sheet paper PCI-HN (14) enabled this approach to be used in a routine clinical setting 
Table 3 Number of PCI items selected by 94 patients using the PCI for the first time, by casemix

\begin{tabular}{|c|c|c|c|c|c|c|}
\hline \multirow[t]{2}{*}{ Characteristics } & \multirow[t]{2}{*}{ Patients } & $\begin{array}{l}\text { Total PCI items } \\
\text { selected }^{*}\end{array}$ & $\begin{array}{c}\text { PCI physical } \\
\& \text { functional } \\
\text { wellbeing domain } \\
\text { items selected* }\end{array}$ & $\begin{array}{l}\text { Cancer treatment } \\
\text { related domain }\end{array}$ & $\begin{array}{l}\text { Social care/social } \\
\text { wellbeing } \\
\text { domain }^{\star \star}\end{array}$ & $\begin{array}{c}\text { Psychological } \\
\text { \& emotional } \\
\text { wellbeing/spiritual } \\
\text { domain }^{\star \star}\end{array}$ \\
\hline & & \multicolumn{2}{|c|}{ Median (IQR) } & \multicolumn{3}{|c|}{$\geq 1$ item selected } \\
\hline Total & 94 & 7 [5-10] & $6[4-8]$ & $38 \%[36]$ & $32 \%[30]$ & $33 \%[31]$ \\
\hline Female & 30 & 7 [5-10] & 6 [4-9] & $47 \%[14]$ & $37 \%[11]$ & $40 \%[12]$ \\
\hline Male & 64 & 7 [5-10] & $6[4-8]$ & $34 \%[22]$ & $30 \%[19]$ & $30 \%[19]$ \\
\hline$P$ value & & 0.57 & 0.76 & 0.27 & 0.64 & 0.35 \\
\hline \multicolumn{7}{|l|}{ Age at baseline } \\
\hline$P$ value & & 0.82 & 0.86 & 0.21 & 0.83 & 0.38 \\
\hline \multicolumn{7}{|l|}{ Tumour site } \\
\hline Oral cavity & 19 & $7[5-11]$ & $4[3-9]$ & $32 \%[6]$ & $63 \%[12]$ & $63 \%[12]$ \\
\hline Oropharynx & 47 & $7[5-10]$ & 6 [4-9] & $43 \%[20]$ & $26 \%[12]$ & $23 \%[11]$ \\
\hline Larynx & 15 & $6[4-9]$ & $5[2-7]$ & $47 \%[7]$ & $27 \%[4]$ & $13 \%[2]$ \\
\hline Other ${ }^{\star \star \star}$ & 13 & $6[3-10]$ & $6[3-8]$ & $23 \%[3]$ & $15 \%[2]$ & $46 \%[6]$ \\
\hline$P$ value & & 0.39 & 0.26 & 0.5 & 0.015 & 0.004 \\
\hline \multicolumn{7}{|l|}{ Overall clinical stage } \\
\hline RT only & 28 & 7 [5-10] & 6 [4-9] & $39 \%[11]$ & $14 \%[4]$ & $21 \%[6]$ \\
\hline RT \& CT only & 41 & $7[4-11]$ & $6[4-9]$ & $39 \%[16]$ & $29 \%[12]$ & $29 \%[12]$ \\
\hline Surgery \& (RT or RT\&CT) & 9 & $6[5-12]$ & $5[4-8]$ & $33 \%[3]$ & $44 \%[4]$ & $56 \%[5]$ \\
\hline$P$ value & & 0.9 & 0.67 & $>0.99$ & 0.002 & 0.06 \\
\hline
\end{tabular}

*, Mann-Whitney (2 comparison groups) or Kruskal-Wallis (>2 groups) test; ${ }^{* *}$, Fishers exact test; ${ }^{* \star}$, parotid [6], metastatic lymph node/ unknown primary [5], follicular lymphoma post nasal space [1], piriform fossa [1]. RT, radiotherapy; CT, chemotherapy; PCI, Patient Concerns Inventory.

without needing access to technology. Furthermore, it promoted a patient led approach to the consultation, in a time constrained clinical situation. This also supports the move to Personalized Stratified Follow Up (PSFU) for cancer patients as an effective way of adapting care to their needs, making services more efficient and cost-effective (19).

The focus of this study was reflections related to the inclusion of the PCI into the assessment of patients post treatment. Most patients found it easy to complete, it caused no problem in the running of appointments, the 


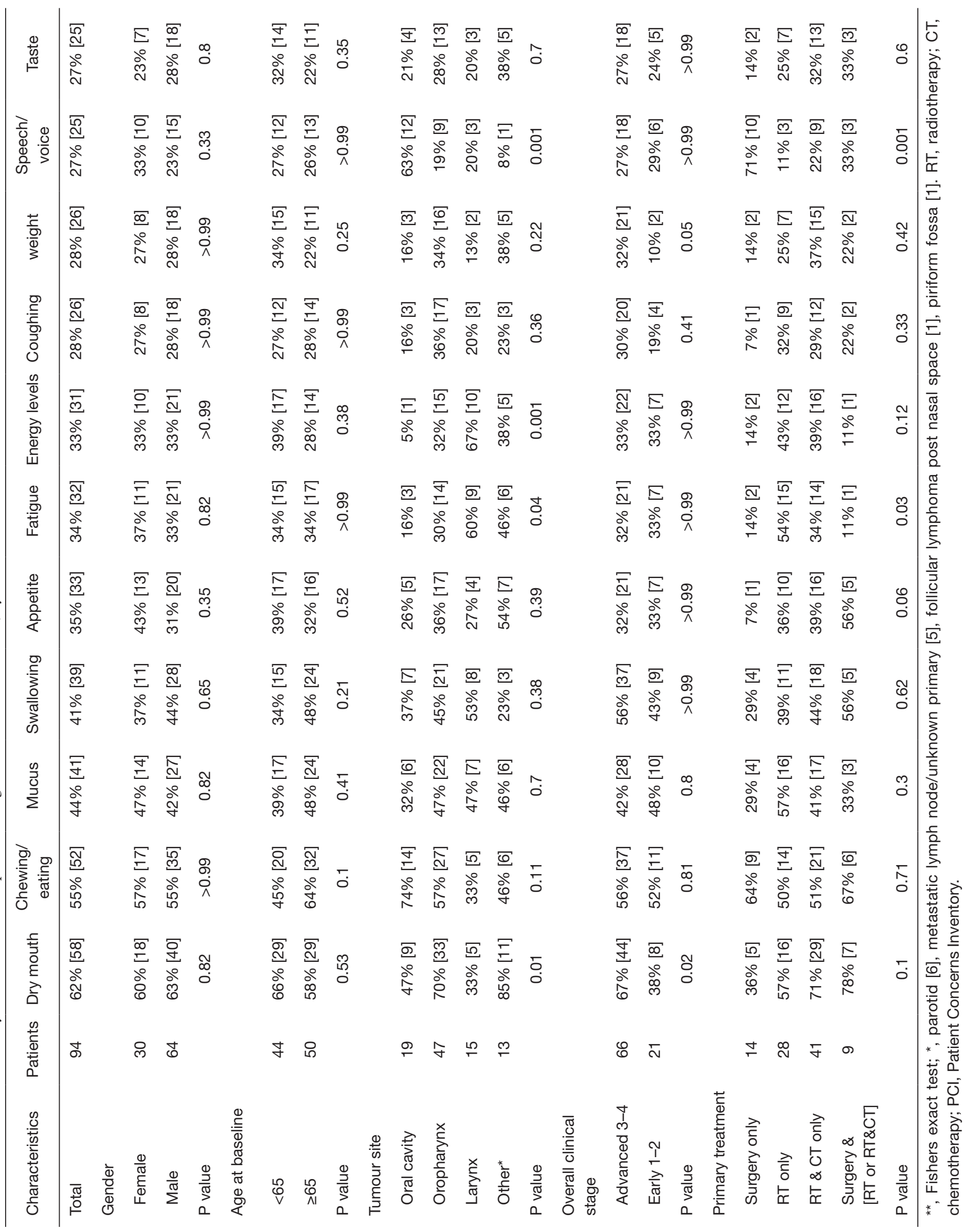




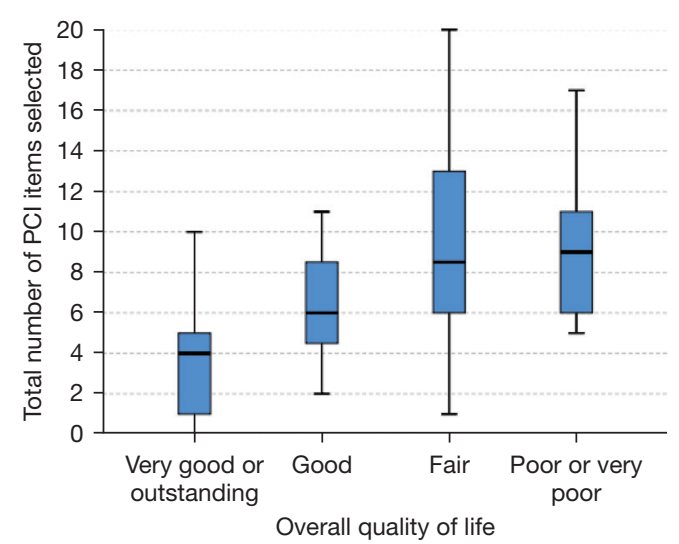

Figure 2 Number of PCI items selected initially, by overall QOL. PCI, percutaneous coronary intervention; QOL, quality of life.

items were discussed, and patients wanted to continue using it. Apart from two patients saying there had been "a little" bit of a problem in the running of their appointments, there were no negative responses.

A wide range of concerns were reported across all the domains (Table 2), suggesting that SLT and Diet have a role in supporting patients' well-being beyond the physical and functional impact of treatment, in collaboration with the wider MDT. The most common selections were dry mouth, chewing/eating, mucus, swallowing, appetite, fatigue/tiredness, energy levels, coughing, weight, speech/ voice/being understood and taste. Difficulties with mucus, chewing/eating, and swallowing are commonly reported post treatment $(15,16)$. Dry mouth and taste changes can also persist and have been found to be significantly associated with oral intake $(16,17)$. Fatigue, energy levels and appetite are commonly reported issues following HNC treatment and can affect eating and drinking $(18,20)$. Studies have found that patients relate their oral intake to fatigue. Fitting meals around sleep schedules is problematic, as some sleep for long periods. Patients outlined a general loss of interest in food, they "couldn't be bothered" with eating, and frequently missed meals. The act of eating, selecting, and preparing altered textures was a chore and effortful, exacerbating fatigue and motivation (21). There is often a relationship between fatigue and mood, and this can affect appetite, nutrition, and weight loss (18). Nutrition is a frequent concern for patients $(22,23)$, and weight loss is independently associated with deterioration in QOL and can have a negative influence on survival (24-26). Patients are often focussed on maintaining or increasing their weight and use it as a barometer of treatment effectiveness. It is often a motivating factor in relation to oral intake when they are unable to derive pleasure from food (21). Speech and voice changes are also common following surgical and oncological treatment and are often the focus of SLT intervention (2).

The concerns raised were broadly similar to other studies $(13,14)$ with a high number of patients reporting issues around dry mouth, chewing/eating, mucus, swallowing, fatigue/tiredness, and speech/voice/being understood. There were, however, some differences. Our study reported a higher frequency of concerns around taste, coughing, appetite, and weight. Taste is often associated with post treatment toxicity, and it can be a barrier to returning to full oral intake $(16,17)$. Coughing may relate to difficulties swallowing, and appetite and weight are often the focus of dietetic consultations in this group. Conversely, in studies where the consultation was with the surgeon, the focus was often on other concerns around fear of the cancer coming back, dental issues, pain in the head and neck and shoulder, cancer treatment and mouth opening $(13,14)$. These are questions which are appropriate to raise with the surgical team who diagnosed and treated the patient. It would therefore seem that patients highlight different items depending on the context of the consultation.

Issues varied to some degree by tumour site and stage (Table 4), but interpretation of this is difficult due to the heterogeneity of the sample with respect to treatment modality. In our study, a higher proportion of patients with oral cavity tumours selected speech/voice issues while a higher proportion treated only by surgery also selected speech/voice issues. These results were confounded because twelve of the nineteen with oral cavity tumours were treated by surgery alone. These patients would be less likely to select issues relating to the side-effects of radiotherapy, while surgery can affect a range of structures involved in speech. Conversely, a higher proportion of patients with tumours located elsewhere and of patients treated by radiotherapy or chemo-radiotherapy selected fatigue and energy level items. These results were also confounded because seventy one out of seventy-three of the patients with tumours in other sites had been treated with radiotherapy or chemo-radiotherapy, and these treatments can have a significant impact on fatigue and energy levels (27). This differs from results in a recent study, which found little association with tumour site while tumour stage seemed more specifically relevant to physical functioning (14). Our study showed no association of tumour stage with selection of speech/voice issues. Other studies found speech/voice issues were more often raised 
by patients with laryngeal tumours than by other patients whilst issues relating to saliva were particularly common for patients with oropharyngeal tumours (13). These studies were carried out in a different setting, with a larger sample of patients, which might explain the difference in responses. There was an association between overall QOL, and the total number of PCI-HN items selected (Figure 2), which is similar to other studies (14). Although the numbers are too small in this study, patient adaptation and coping might be evidenced through the change in overall QOL over time in the 62 patients using the PCI-HN a second time with the percentage reporting a good or better QOL increasing from $47 \%$ to $66 \%$.

Patients were offered a range of advice and interventions in relation to the concerns they raised. The involvement of the wider MDT is essential in providing a high standard of patient care in this group, and a number of concerns required onward referral and signposting to different specialist professionals within the team. Patients reporting fatigue/tiredness and energy levels were signposted to the Macmillan Get Active/Feel Good service. Patients reporting concerns around mucus and dry mouth were referred to the Dental Hygienist for review. A number of patients also reported concerns around issues such as fear of the cancer coming back, depression, mood, and anxiety, and were offered referral on for psychological support and counselling. Issues relating to shoulder function were addressed by referral to Physiotherapy. Concerns around taste, chewing/eating, swallowing, coughing, speech/voice/ being understood, appetite, weight, and PEG tube, were addressed by the appropriate SLT and Diet clinicians. Advice was provided around ways to help, and what to expect in terms of improvement. Interventions were also delivered around techniques and strategies to deal with these, and the SLT identified patients who would benefit from further objective instrumental assessments. Patients reported that the PCI-HN helped communication with the clinician.

This study has a number of limitations. The sample was heterogeneous, in terms of tumour size, location, and treatment schedule. However, the proportion of HNC sites reflected the cancer incidence and workload of the clinic, with half being oropharyngeal. A larger sample would have allowed more specific comment on case mix factors and possible interactions. Study recruitment and follow-up ended in March 2020 because of the COVID-19 outbreak, with a reduction in face to face consultations and a move to the use of telehealth. Unfortunately, there was no capacity to administer the questionnaire in this setting. This limited the sample size and the repeat longitudinal element of the study. Data in this current study were collected without additional funding. The evaluation questionnaire was only incorporated part way through the study, due to staffing issues, thus, data were limited. Administration depended on clinic capacity and support staff availability, resulting in some missing PCI-HN data. Although our data suggest that the use of the PCI-HN can be beneficial to the consultation, the evaluation form was not anonymised, and this may have influenced the results. Furthermore, there was no measure of the clinicians' experience, or whether it had any impact on the discussion or recommendations. The clinics function in a very similar way, but it is recognised that in a more indepth study the inclusion of a measure of fidelity to assess any differences in approach and use of the PCI would be beneficial. The study data were limited to two clinics in one centre, and the findings might not be generalizable to similar clinics at other centres, which may have a different patient case mix. The study focused on reporting the completion of the one-page PCI-HN sheet by patients, and no attempt was made to incorporate objective outcomes or other patient reported questionnaires. Also, the findings are considered exploratory given that there is no comparison as to what is achieved by the questionnaire, no control group or other alternative and no additional outcome measure to demonstrate how the prompt list was beneficial.

The heterogeneous sample in this study was adequate in this context, however, it would be valuable to collect more data to support the use of the PCI-HN in this clinic setting, which could be applied to specific patient groups. Since the COVID-19 outbreak, there has been a recent shift to the use of remote consultations, and studies are currently looking at the use of PCI-HN during the COVID-19 pandemic $(28,29)$. These may be valuable areas for further research in our clinics. Although added late in the study, the evaluation questionnaire provided valuable preliminary data suggesting benefits from using the PCI-HN in the SLT/Diet setting. Identifying both the impairment, and psychosocial consequences, of communication and eating and drinking difficulties in HNC survivors, is key to supporting patients on their recovery journey. A PCI-HN approach in routine follow-up clinics is being evaluated for a clinically meaningful benefit in QOL and the domains of UW-QOL at 1 year and is the subject of ongoing research (11).

\section{Conclusions}

Our preliminary data suggest that the PCI-HN is suitable 
for use in routine clinical practice in combined SLT and Diet clinics, as an adjunct to routine clinical care. It helps raise some of the wider issues that could otherwise be missed and is a suitable assessment tool specific to the swallowing, communication and nutrition needs of patients with $\mathrm{HNC}$, post treatment. Early detection of issues which impact on eating, drinking and communication, allows the opportunity for the identification, and provision, of SLT and Dietetic interventions, which can aid adaption, support patients through their recovery, and ultimately, promote better outcomes.

\section{Acknowledgments}

With thanks to Katie McLaughlin, Lauren Burch, and Carol Glaister for their help with data collection, and Clare Probert for her comments on the manuscript.

Funding: None.

\section{Footnote}

Provenance and Peer Review: This article was commissioned by the editorial office, Frontiers of Oral and Maxillofacial Medicine for the series "Health-Related-Quality of Life in Head and Neck Oncology". The article has undergone external peer review.

Reporting Checklist: The authors have completed the STROBE reporting checklist. Available at https://fomm. amegroups.com/article/view/10.21037/fomm-21-81/rc

Data Sharing Statement: Available at https://fomm. amegroups.com/article/view/10.21037/fomm-21-81/dss

Conflicts of Interest: All authors have completed the ICMJE uniform disclosure form (available at https://fomm. amegroups.com/article/view/10.21037/fomm-21-81/coif). The series "Health-Related-Quality of Life in Head and Neck Oncology" was commissioned by the editorial office without any funding or sponsorship. SNR served as the unpaid Guest Editor of the series. The authors have no other conflicts of interest to declare.

Ethical Statement: The authors are accountable for all aspects of the work in ensuring that questions related to the accuracy or integrity of any part of the work are appropriately investigated and resolved. The study was conducted in accordance with the Declaration of Helsinki (as revised in 2013). The data for this study collected were collected during routine follow up HNC clinics. The present study was granted local audit approval by the Shrewsbury and Telford Hospital Trust audit department but did not require formal IRAS ethics approval. Informed consent was taken from all individual participants.

Open Access Statement: This is an Open Access article distributed in accordance with the Creative Commons Attribution-NonCommercial-NoDerivs 4.0 International License (CC BY-NC-ND 4.0), which permits the noncommercial replication and distribution of the article with the strict proviso that no changes or edits are made and the original work is properly cited (including links to both the formal publication through the relevant DOI and the license). See: https://creativecommons.org/licenses/by-nc-nd/4.0/.

\section{References}

1. Handle-on-QOL-Quality of life in head and neck cancer. Available online: http://www.handle-on-qol.com.

2. Clarke P, Radford K, Coffey M, et al. Speech and swallow rehabilitation in head and neck cancer: United Kingdom National Multidisciplinary Guidelines. J Laryngol Otol 2016;130:S176-80.

3. Nguyen NA, Ringash J. Head and neck cancer survivorship care: a review of the current guidelines and remaining unmet needs. Curr Treat Options Oncol 2018;19:44.

4. Jacobi I, van der Molen L, Huiskens H, et al. Voice and speech outcomes of chemoradiation for advanced head and neck cancer: a systematic review. Eur Arch Otorhinolaryngol 2010;267:1495-505.

5. Greco E, Simic T, Ringash J, et al. Dysphagia treatment for patients with head and neck cancer undergoing radiation therapy: a meta-analysis review. Int J Radiat Oncol Biol Phys 2018;101:421-44.

6. Ghazali N, Kanatas A, Scott B, et al. Use of the Patient Concerns Inventory to identify speech and swallowing concerns following treatment for oral and oropharyngeal cancer. J Laryngol Otol 2012;126:800-8.

7. Shunmugasundaram C, Rutherford C, Butow PN, et al. Content comparison of unmet needs self-report measures used in patients with head and neck cancer: A systematic review. Psychooncology 2019;28:2295-306.

8. Rogers SN, El-Sheikha J, Lowe D. The development of a Patients Concerns Inventory (PCI) to help reveal patients concerns in the head and neck clinic. Oral Oncol 
2009;45:555-61.

9. Ghazali N, Kanatas A, Bekiroglu F, et al. The patient concerns inventory: a tool to uncover unmet needs in a cancer outpatient clinic. Bull Roy Coll Surg Engl 2013;95:1-6.

10. Allen S, Lowe D, Harris RV, et al. Is social inequality related to different patient concerns in routine oral cancer follow-up clinics? Eur Arch Otorhinolaryngol 2017;274:451-9.

11. Rogers SN, Allmark C, Bekiroglu F, et al. Improving quality of life through the routine use of the patient concerns inventory for head and neck cancer patients: baseline results in a cluster preference randomised controlled trial. Eur Arch Otorhinolaryngol 2020;277:3435-47.

12. Rogers SN, Ahiaku S, Lowe D. Is routine holistic assessment with a prompt list feasible during consultations after treatment for oral cancer? Br J Oral Maxillofac Surg 2018;56:24-8.

13. Kanatas A, Ghazali N, Lowe D, et al. Issues patients would like to discuss at their review consultation: variation by early and late stage oral, oropharyngeal and laryngeal subsites. Eur Arch Otorhinolaryngol 2013;270:1067-74.

14. Rogers SN, Alvear A, Anesi A, et al. Variations in concerns reported on the patient concerns inventory in patients with head and neck cancer from different health settings across the world. Head Neck 2020;42:498-512.

15. Wilson JA, Carding PN, Patterson JM. Dysphagia after nonsurgical head and neck cancer treatment: patients' perspectives. Otolaryngol Head Neck Surg 2011;145:767-71.

16. Kamal M, Barrow MP, Lewin JS, et al. Modeling symptom drivers of oral intake in long-term head and neck cancer survivors. Support Care Cancer 2019;27:1405-15.

17. Zuydam AC, Rogers SN, Grayson K, et al. Routine use of swallowing outcome measures following head and neck cancer in a multidisciplinary clinic setting. Int Arch Otorhinolaryngol 2021;25:e185-92.

18. Röing M, Hirsch JM, Holmström I. The uncanny mouth a phenomenological approach to oral cancer. Patient Educ Couns 2007;67:301-6.

19. Implementing personalised stratified follow-up pathways. NHS England and NHS Improvement. March 2020.

20. Molassiotis A, Rogers M. Symptom experience and regaining normality in the first year following a diagnosis of head and neck cancer: a qualitative longitudinal study. Palliat Support Care 2012;10:197-204.

21. Patterson JM, McColl E, Wilson J, et al. Head and neck cancer patients' perceptions of swallowing following chemoradiotherapy. Support Care Cancer 2015;23:3531-8.

22. Larsson M, Hedelin B, Athlin E. Lived experiences of eating problems for patients with head and neck cancer during radiotherapy. J Clin Nurs 2003;12:562-70.

23. Shragge JE, Wismer WV, Olson KL, et al. Shifting to conscious control: psychosocial and dietary management of anorexia by patients with advanced cancer. Palliat Med 2007;21:227-33.

24. Langius JA, van Dijk AM, Doornaert P, et al. More than $10 \%$ weight loss in head and neck cancer patients during radiotherapy is independently associated with deterioration in quality of life. Nutr Cancer 2013;65:76-83.

25. Jager-Wittenaar H, Dijkstra PU, Vissink A, et al. Malnutrition and quality of life in patients treated for oral or oropharyngeal cancer. Head Neck 2011;33:490-6.

26. Ravasco P, Monteiro-Grillo I, Marques Vidal P, et al. Impact of nutrition on outcome: a prospective randomized controlled trial in patients with head and neck cancer undergoing radiotherapy. Head Neck 2005;27:659-68.

27. Bossi P, Di Pede P, Guglielmo M, et al. Prevalence of Fatigue in Head and Neck Cancer Survivors. Ann Otol Rhinol Laryngol 2019;128:413-9.

28. Kanatas A, Rogers SN. The role of the head and neck cancer-specific patient concerns inventory (PCI-HN) in telephone consultations during the COVID-19 pandemic. Br J Oral Maxillofac Surg 2020;58:497-9.

29. Kanatas A, Rogers SN. The after diagnosis head and neck cancer-specific patient concerns inventory (HaNC$\mathrm{AD}$ ) as a pre-treatment preparation aid during the COVID-19 pandemic. Eur Arch Otorhinolaryngol 2020;277:2141-5.

doi: 10.21037/fomm-21-81

Cite this article as: Zuydam AC, Lowe D, Rogers SN. Evaluation of the head and neck cancer patient concerns inventory in a cohort of patients attending routine multidisciplinary speech and language therapy/dietitian follow up clinics. Front Oral Maxillofac Med 2022;4:3. 


\section{Supplementary}

Table S1 Overall QOL by case mix

\begin{tabular}{|c|c|c|c|c|c|c|c|}
\hline Characteristics & Patients & $\begin{array}{l}\text { Overall QOL less than } \\
\text { good on first occasion }\end{array}$ & $\begin{array}{l}\text { With QOL on first and } \\
\text { second occasion }\end{array}$ & \multicolumn{4}{|c|}{ Change in overall QOL } \\
\hline Total & 91 & $47 \%[43]$ & $68 \% 62 / 91$ & 17 & 16 & 4 & 25 \\
\hline \multicolumn{8}{|l|}{ Gender } \\
\hline Female & 29 & $38 \%[11]$ & $62 \% 18 / 29$ & 4 & 3 & 3 & 8 \\
\hline$P$ value & & 0.26 & & \multicolumn{4}{|c|}{0.18} \\
\hline \multicolumn{8}{|l|}{ Age at baseline } \\
\hline$<65$ & 43 & $49 \%[21]$ & $72 \% 31 / 43$ & 9 & 8 & 1 & 13 \\
\hline$\geq 65$ & 47 & $45 \%[21]$ & $64 \% 30 / 47$ & 8 & 7 & 3 & 12 \\
\hline Oral cavity & 19 & $47 \%[9]$ & $63 \% 12 / 19$ & 5 & 2 & 1 & 4 \\
\hline Oropharynx & 46 & $50 \%[23]$ & $72 \% 33 / 46$ & 8 & 9 & 1 & 15 \\
\hline Larynx & 15 & $47 \%[7]$ & $60 \% 9 / 15$ & 2 & 3 & 0 & 4 \\
\hline Other & 11 & $36 \%[4]$ & $73 \% 8 / 11$ & 2 & 2 & 2 & 2 \\
\hline$P$ value & & 0.9 & & \multicolumn{4}{|c|}{0.64} \\
\hline \multicolumn{8}{|l|}{ Overall clinical stage } \\
\hline Advanced 3-4 & 65 & $46 \%[30]$ & $71 \% 46 / 65$ & 13 & 11 & 2 & 20 \\
\hline Early $1-2$ & 21 & $48 \%[10]$ & $52 \% 11 / 21$ & 2 & 4 & 0 & 5 \\
\hline Surgery \& (RT or RT\&CT) & 9 & $56 \%[5]$ & $67 \% 6 / 9$ & 3 & 1 & 0 & 2 \\
\hline$P$ value & & 0.74 & & \multicolumn{4}{|c|}{0.94} \\
\hline
\end{tabular}

LL, less than good on first and second occasions; LG, less than good on first, good or better on second; GL, good or better on first, less than good on second; GG, good or better on both occasions; QOL, quality of life. 